\title{
Optimal representations of quantum states by gaussians in phase space
}

\author{
Anatole Kenfack $\uparrow$ Jan M Rost $\uparrow$ and Alfredo M Ozorio de \\ Almeida $\$$ \\ $\dagger$ Max Planck Institute for the Physics of Complex Systems Nöthnitzer Strasse 38, \\ 01187 Dresden, Germany \\ $\ddagger$ Centro Brasileiro de Pesquisas Fisicas, R Xavier Sigaud 150, 22290-180 Rio de \\ Janeiro, Brazil
}

\begin{abstract}
A two-step optimization is proposed to represent an arbitrary quantum state to a desired accuracy with the least number of gaussians in phase space. The Husimi distribution of the quantum state provides the information to determine the modulus of the weight for the gaussians. Then, the phase information contained in the Wigner distribution is used to obtain the full complex weights by considering the relative phases for pairs of gaussians, the chords. The method is exemplified with several excited states $n$ of the harmonic and the Morse oscillators. A semiclassical interpretation of the number of gaussians needed as a function of the quantum number $n$ is given. The representation can also be used to characterize Wigner and Husimi distributions directly which do not originate in a quantum state.
\end{abstract}

$\S$ To whom correspondence should be addressed (kenfack@mpipks-dresden.mpg.de) 


\section{Introduction}

In many situations there is a need to represent a given quantum state or a probability distribution of states in terms of a simple basis which can be easily handled in further processing, e.g., for integration in matrix elements. Gaussians form such a basis although it is overcomplete. Gaussians have the additional advantage that they can adopt the form of coherent states in phase space, thereby supplying a direct link to classical mechanics. Hence, the formulation in terms of gaussians is also very useful for a mixed quantum-classical or quasiclassical description of a system. In particular the evolution of systems with many degrees of freedom can often be described within classical molecular dynamics only. Here, one is interested in obtaining optimal initial conditions by translating the initial quantum state of the system into a suitable classical phase space distribution which can be classically propagated [1. While all quantum information on an amplitude level is lost in this approximation, we will introduce a representation which can preserve basic quantum interferences by construction even in the case of the purely classical propagation. Finally, it is of general and basic interest to reconstruct amplitudes or the density matrix, i.e., coherent information, from phase space distributions. This has been done recently in terms of the moments in time of the Wigner distribution [2]. Although there is no principal need to do so we will concentrate on the Wigner distribution due to the attention it has received recently in different areas. In several experiments the Wigner distribution of microscopic systems has been measured [3]. Beyond the quantum context, in the time-frequency phase space of signal processing, analysis of the Wigner distribution [4] is an important tool [4], as for optics [5], speech analysis [6] or the monitoring of machinery conditions [7].

The decomposition of a given state $|\Psi\rangle$ into coherent states can be regarded as a particular form of phase space representation. Labeling positions by $q$ or $Q$ and momenta by $p$ or $P$, we translate the ground state of the harmonic oscillator by $X=(P, Q)$ to obtain the coherent state in position representation, i.e. its wavefunction is [8, 11]

$$
\langle q \mid X\rangle=\left(\frac{m \omega}{\pi \hbar}\right)^{1 / 4} \exp \left(-\frac{\omega}{2 \hbar}(q-Q)^{2}+i \frac{P}{\hbar}\left(q-\frac{Q}{2}\right)\right) .
$$

For simplicity, we chose unit frequency $(\omega=1)$ and mass $(m=1)$ for the harmonic oscillator without loss of generality. (Often the complex variable $Z=(Q+i P) / \sqrt{\hbar}$ is used and the constant phase $-P Q / 2 \hbar$ is omitted). Even though the coherent state basis is overcomplete, the exact representation,

$$
|\Psi\rangle=\frac{1}{\pi} \int d X|X\rangle\langle X \mid \Psi\rangle
$$

is unique [8, 11]. However, such a decomposition into coherent states is monstrously inefficient, as shown by the overlap of coherent states,

$$
\begin{aligned}
\left\langle X \mid X^{\prime}\right\rangle & =\exp \left(-\frac{\left(Q-Q^{\prime}\right)^{2}}{4 \hbar}-\frac{\left(P-P^{\prime}\right)^{2}}{4 \hbar}+\frac{i}{2 \hbar}\left(Q P^{\prime}-Q^{\prime} P\right)\right) \\
& \equiv \exp \left(-\frac{\left(X-X^{\prime}\right)^{2}}{4 \hbar}+\frac{i}{2 \hbar} X \wedge X^{\prime}\right)
\end{aligned}
$$


so that

$$
\left|\left\langle X \mid X^{\prime}\right\rangle\right|^{2}=\exp \left(-\frac{\left(X-X^{\prime}\right)^{2}}{2 \hbar}\right) .
$$

We have used the wedge product,

$$
X \wedge X^{\prime}=P Q^{\prime}-Q P^{\prime}=(\mathbf{J} X) \cdot X^{\prime},
$$

to shorten the notation. (With the second equation we also define the symplectic matrix $\mathbf{J})$. It follows that one can approximate any given state by a finite expansion

$$
|\Psi\rangle \cong \sum_{j} a_{j}\left|X_{j}\right\rangle
$$

with arbitrary accuracy. One possibility would be to place the coherent states on a grid and then determine the complex coefficients $a_{j}$ by minimizing the error, defined in some convenient way. Of course, it is much better to use some features of $|\Psi\rangle$ as a preliminary guide to where the $\left|X_{j}\right\rangle$ should be located.

An obvious necessary condition for a good fit is that the coherent state intensity $|\langle X \mid \Psi\rangle|^{2}$ in (4) be well approximated by

$$
\begin{aligned}
|\langle X \mid \Psi\rangle|^{2} & =\left|\sum_{j} a_{j}\left\langle X \mid X_{j}\right\rangle\right|^{2} \\
& =\sum_{j}\left|a_{j}\right|^{2}\left|\left\langle X \mid X_{j}\right\rangle\right|^{2}+\sum_{j \neq k} a_{j} a_{k}^{*}\left\langle X \mid X_{j}\right\rangle\left\langle X \mid X_{k}\right\rangle^{*}
\end{aligned}
$$

for each phase space point $X$. But note that, if the diagonal terms in this expression are only appreciable for $X_{j}$ very close to $X$, a nondiagonal term decays as a product of gaussians centered on a pair of separate phase space vectors. Such a term is negligible for all $X$ unless $X_{j}$ is very close to $X_{k}$. Moreover, such nondiagonal terms are not positive definite, which justifies our performing the preliminary fit

$$
\rho_{\Psi}(X)=\frac{1}{\pi}|\langle X \mid \Psi\rangle|^{2} \approx \sum_{j}\left|a_{j}\right|^{2} \frac{1}{\pi}\left|\left\langle X \mid X_{j}\right\rangle\right|^{2}=\sum_{j}\left|a_{j}\right|^{2} G_{j}(X),
$$

where

$$
G_{j}(X)=\exp \left(-\frac{\left(X-X_{j}\right)^{2}}{2 \hbar}\right) .
$$

The factor $1 / \pi$ has here been introduced so that the left hand side becomes the Husimi function [9], i.e., the Husimi representation of the state $|\Psi\rangle$, also known as the $Q$-function in quantum optics [11]. Here, $\rho_{\Psi}$ is then decomposed into gaussians phase space packets (gpps) which are Husimi representations of the coherent states, specified by (44).

The Husimi function in its own right uniquely defines a quantum state, though part of the quantum phase information is locked up into its delicate analytic properties. These are hard to translate into other representations, but we propose to sidestep this problem. Our method is to make a preliminary fit of the Husimi function (8), which is real and positive definite, and therefore much simpler than fitting the complex coherent states directly in (6). However we can now identify the coefficients of the Husimi fitting as being proportional to the square modulus of the coefficients of the superposition of 
coherent states (6). The phases of the coefficients are then determined by relating the Husimi function to the coarse-graining of the Wigner function, i.e. we bring in yet a third phase space representation of the state $|\Psi\rangle$. It should be pointed out that, once the state $|\Psi\rangle$ has been fitted by a finite number of coherent states, it can be viewed in any representation. The special attention given here to the Wigner function is justified by the way that it highlights quantum interferences. In fact such interferences are sometimes mimicked by extra gaussians in the context of numerical work with classical densities [1]. Thus, in the next section we present the Wigner function, its relation to the Husimi function and its specific form for an arbitrary superposition of gaussians. In addition, we discuss and establish the differences between the representation of quantum states such as the wavefunction, the Wigner distribution and the Husimi distribution using these gaussians. The Wigner distribution sets the stage for the delicate procedure of fitting the phases, i.e., to determine the appropriate interference fringes between the gaussians as detailed in section 3. In this section we propose a method for determining the phase of the expansion coefficients by Fourier analysing the Wigner function. Actually, this resulting chord function or characteristic function (in quantum optics [10]) can be derived directly from the initial wave function, without obtaining first the Wigner function. The Husimi function, on the other hand, supplies a robust overall picture of the quantum states which can be used to place the gaussians. This will be discussed in section 4 using explicit examples for illustration. Emphasis will be put on general symmetry criteria for placing the gaussians and numerical demonstrations based on Monte Carlo fitting of sampled wavefunctions. Finally in section 5, we semiclassically interpret the results of section 4, namely the increase of gaussians needed to represent quantum states $\psi_{n}$ of a system with increasing quantum number $n$. A summary in section 6 concludes the paper.

For simplicity, all our formulae are presented for systems with a single degree of freedom, but they are easily generalized to high dimensional systems.

\section{Wigner functions and their relations}

The state $|\Psi\rangle$ is uniquely described by the real function of phase space points $x=(p, q)$,

$$
W_{\Psi}(x)=\int \frac{d q^{\prime}}{2 \pi \hbar}\left\langle q+\frac{q^{\prime}}{2} \mid \Psi\right\rangle\left\langle\Psi \mid q-\frac{q^{\prime}}{2}\right\rangle \exp \left(-\frac{i}{\hbar} p q^{\prime}\right)
$$

known as the Wigner function [12. Inversion of this symmetrized Fourier transform leads back to the wavefunction, while its intensity results from the projection

$$
|\langle q \mid \Psi\rangle|^{2}=\int d p W_{\Psi}(x)
$$

Though the Wigner function can be negative for some points $x$, its projection is always positive. Actually, this is just a particular case of the general relation,

$$
|\langle\Phi \mid \Psi\rangle|^{2}=2 \pi \hbar \int d x W_{\Phi}(x) W_{\Psi}(x)
$$


for arbitrary states $|\Phi\rangle$ and $|\Psi\rangle$. Thus, introducing the Wigner function for the coherent state $|X\rangle$,

$$
W_{X}(x)=\frac{1}{\pi \hbar} \exp \left(-\frac{(x-X)^{2}}{\hbar}\right),
$$

we obtain the Husimi representation of $|\Psi\rangle$ as

$$
\begin{aligned}
\frac{1}{\pi}|\langle X \mid \Psi\rangle|^{2} & =2 \hbar \int d x W_{X}(x) W_{\Psi}(x) \\
& =\frac{2}{\pi} \int d x \exp \left(-\frac{(x-X)^{2}}{\hbar}\right) W_{\Psi}(x) .
\end{aligned}
$$

This shows that the positive definite Husimi function results from the gaussian smoothing of the Wigner function. Inserting the coherent state expansion $|\Psi\rangle$ given by (6) into the Wigner transform (10), we obtain the well known form of the corresponding Wigner function,

$$
W_{\Psi}(x)=\sum_{j}\left|a_{j}\right|^{2} W_{X_{j}}+2 \operatorname{Re}\left(\sum_{j \neq k} a_{j} a_{k}^{*} W_{j k}(x)\right),
$$

where we define the crossed Wigner function (or Moyal bracket [13])

$$
\begin{aligned}
W_{j k}(x) & \equiv \int \frac{d q^{\prime}}{2 \pi \hbar}\left\langle q+\frac{q^{\prime}}{2} \mid X_{j}\right\rangle\left\langle X_{k} \mid q-\frac{q^{\prime}}{2}\right\rangle \exp \left(-\frac{i}{\hbar} p q^{\prime}\right) \\
& =\frac{1}{\sqrt{2} \pi \hbar} \exp \left(-\frac{\left(x-\bar{X}_{j k}\right)^{2}}{\hbar}\right) \exp \left(-\frac{i}{\hbar}\left(x \wedge \delta X_{j k}+\frac{1}{2} X_{k} \wedge X_{j}\right)\right) .
\end{aligned}
$$

Here we use the abbreviated relations $\bar{X}_{j k}=\left(X_{k}+X_{j}\right) / 2$ and $\delta X_{j k}=X_{j}-X_{k}$, as well as the skew product (15). Comparing (15) with (7) we find the same gaussian form for the diagonal terms of the Wigner function as for the Husimi function, but the offdiagonal terms are no longer negligible. For the Wigner function, any pair of coherent states determines a third gaussian centered halfway between their two centres, of comparable amplitude to the diagonal contributions of the individual gaussians. This interference term is only cancelled by the smoothing employed to obtain the Husimi function, because of the phase oscillations. Their wavelengths in phase space are $2 \pi \hbar /|\delta X|$, so that the Husimi function is increasingly damped with growing separation of these gpps. This is an excellent illustration of the way that the Husimi function highlights the classical structure in a quantum state, while it hides quantum phase information. This question will be further developed in section 4. Of course the phase information is still contained in the small offdiagonal terms in (7), because the Husimi function is fully quantum mechanical, but this information is certainly more accessible in the Wigner representation.

We can now understand the differences between using the position representation, i.e. the wave function, the Wigner representation or the Husimi representation, to fit a given state by a set of coherent states. The position representation has the advantage that there are no interference terms between pairs of coherent states. However, if the state is highly oscillatory, such as a highly excited eigenstate of a Hamiltonian, this 
needs to be fitted by coherent states with large momenta $P$, which also have narrow oscillations. Clearly the fitting procedure becomes very unstable with respect to small errors in the initial function if large momenta are necessary. In contrast, the diagonal terms in the expansion of the Wigner function are smooth gaussians distributed over phase space, but again we obtain increasingly narrow oscillations for the interferences around the midpoint of each pair. Of course, the oscillations of the wave function and the Wigner function are not unrelated, since the latter have the same wavelength as the wave intensity in the case of a pair of gaussians located at $( \pm P, Q)$.

Finally, the Husimi function washes out oscillations, the more effectively the tighter they happen to be. We shall review in section 4 how the smooth phase space distribution singles out the basic classical structure within the quantum state, which can be stably fitted by real gpps.

The cost is that only the location of the gaussians in phase space and the square modulus of their coefficients are then determined. In the next section we present a method for retrieving the phases of the coefficients by taking the Fourier transform of the Wigner function.

\section{Fitting the phases}

Both, the direction and the wavelength of the fringes that modulate the interference peak halfway between two gpps at $X_{j}$ and $X_{k}$ in the Wigner representation, are entirely determined by $\delta X_{j k}$. However, these fringes are translated by changing the phases of the coefficients $a_{j}$ and $a_{k}$, which we wish to determine. Clearly, the best way to recover these phases is to take the Fourier transform of the Wigner function:

$$
\begin{aligned}
\tilde{W}_{\Psi}(\xi) & =\int \frac{d p d q}{2 \pi \hbar} W_{\Psi}(p, q) \exp \left(\frac{i}{\hbar}\left(p \xi_{q}-q \xi_{p}\right)\right) \\
& =\int \frac{d x}{2 \pi \hbar} W_{\Psi}(x) \exp \left(\frac{i}{\hbar}(x \wedge \xi)\right) .
\end{aligned}
$$

It is also possible to express this directly from the wave function

$$
\tilde{W}_{\Psi}(\xi)=\int d q\left\langle q+\frac{\xi_{q}}{2} \mid \Psi\right\rangle\left\langle\Psi \mid q-\frac{\xi_{q}}{2}\right\rangle \exp \left(-\frac{i}{\hbar} \xi_{p} q\right)
$$

which resembles the Wigner transform (10). This function is known as the Woodward ambiguity function in communications theory [14] or the characteristic function in quantum optics [10]. Usually the sign of $\xi_{p}$ is reversed in the definition of $\tilde{W}$, but here we follow [15, where it is simply called the chord function. A justification for this term appears immediately if we consider the case of a superposition of gaussians that is our concern :

$$
\tilde{W}_{\Psi}(\xi)=\sum_{j}\left|a_{j}\right|^{2} \tilde{W}_{j j}(\xi)+\sum_{j \neq k} a_{j} a_{k}^{*}\left(\tilde{W}_{j k}(\xi)+\tilde{W}_{k j}(\xi)\right)
$$

where

$$
\tilde{W}_{j j}(\xi)=\frac{1}{2 \pi \hbar} \exp \left(-\frac{\xi^{2}}{\hbar}+\frac{i}{\hbar} X_{j} \wedge \xi\right)
$$


and

$$
\begin{aligned}
\tilde{W}_{j k}(\xi) & =\frac{1}{2 \sqrt{2} \pi \hbar} \exp \left(-\frac{\left(\xi-\delta X_{j k}\right)^{2}}{4 \hbar}\right) \\
& \times \exp \left(\frac{i}{\hbar}\left(\left(\xi-\delta X_{j k}\right) \wedge \bar{X}_{j k}+\frac{X_{k} \wedge X_{j}}{2}\right)\right) .
\end{aligned}
$$

Thus all the diagonal terms collapse onto gaussians centered on $\xi=0$, whereas each interference term is a gaussian centered on the chord joining $X_{j}$ and $X_{k}$. (Both directions $\pm \delta X_{j k}$ are present, leading to symmetric contributions in chord space. Indeed, we must have $\left.\tilde{W}(-\xi)=\tilde{W}^{*}(\xi)\right)$. Let us now suppose that a given chord $\delta X_{j k}$ is sufficiently far from all the other chords, so that at $\xi=\delta X_{j k}$ the chord function is dominated by the single nondiagonal contribution $\tilde{W}\left(\delta X_{j k}\right)$, i.e.

$$
\begin{aligned}
\tilde{W}_{\Psi}\left(\delta X_{j k}\right)-\sum_{l}\left|a_{l}\right|^{2} \tilde{W}_{l l}\left(\delta X_{j k}\right) & \approx a_{j} a_{k}^{*} \tilde{W}_{j k}\left(\delta X_{j k}\right) \\
& =\frac{a_{j} a_{j}^{*}}{2 \sqrt{2} \pi \hbar} \exp \left(\frac{i}{\hbar} \frac{X_{k} \wedge X_{j}}{2}\right) .
\end{aligned}
$$

Then, defining

$$
a_{j}=\left|a_{j}\right| \exp \left(i \theta_{j}\right)
$$

and recalling that we have already fitted $\left|a_{j}\right|$ from the Husimi function, we obtain $\theta_{j k}=\theta_{j}-\theta_{k}$ from

$$
\theta_{j k}-\frac{1}{2 \hbar} X_{j} \wedge X_{k}=\operatorname{phase}\left(\tilde{W}_{\Psi}\left(\delta X_{j k}\right)-\sum_{l}\left|a_{l}\right|^{2} \tilde{W}_{l l}\left(\delta X_{j k}\right)\right) .
$$

Of course, the modulus of both sides of (22) should be approximately equal, which provides a check on the previous Husimi fitting. The diagonal sum that is substracted from $\tilde{W}_{\Psi}\left(\delta X_{j k}\right)$ in (22) decays exponentially with $\delta X_{j k}^{2}$, so it will only affect the phases of the smaller chords. It might appear to be only consistent with our previous approximation of the Husimi function to neglect the diagonal sum in (22), but this would perturb the phases of small chords.

\section{Placing the gaussians: examples}

So far, nothing has been said about how to place the gaussians in phase space that are meant to approximate a given Husimi function. An obvious criterion is that any relative maximum of this smooth distribution should also receive a coherent state. Further knowledge of the state should also be used. For instance, if it is known to be the eigenstate of a given Hamiltonian $\hat{H}$ with energy $E_{n}$, then the semiclassical considerations in the following section allow the restriction of the gaussian centers to $\left|H(X)-E_{n}\right| \leq \hbar$. Of course, the fitting procedure is only useful for cases in which $\hat{H}$ is unknown, or else $|\Psi\rangle$ may itself be a linear superposition of states, such as result from a two slit experiment. Here we immediately feel the advantage that the decomposition (6) is defined in terms of the state itself, rather than the density operator $|\Psi\rangle\langle\Psi|$. Thus, the linearity of $|\Psi\rangle=a_{1}\left|\Psi_{1}\right\rangle+a_{2}\left|\Psi_{2}\right\rangle$ allows us to fit $\left|\Psi_{1}\right\rangle$ and $\left|\Psi_{2}\right\rangle$ independently and then 
to superpose the fitted state as $|\psi\rangle=a_{1}\left|\psi_{1}\right\rangle+a_{2}\left|\psi_{2}\right\rangle$. A further simplication results from possible symmetries. For instance, for the eigenstate of an even potential, one should place the gaussians symmetrically at $\left(P_{j}, \pm Q_{j}\right)$ with $a_{j-}= \pm a_{j+}$, so as to guarantee even or odd states. Again, this is a very particular case, but time reversal invariance is much more common. If the state $|\Psi\rangle$ resulted from initial real wave functions and the evolution proceeded through interactions that preserve time reversal symmetries (in usual practice, if there are no magnetic fields) then the final state can also be real. In this case, one must choose symmetric pairs of gaussians at $\left( \pm P_{j}, Q_{j}\right)$ with equal real coefficients, $a_{j-}=a_{j+}$, so as to ensure that $|\Psi\rangle$ is also real. This halves the number of independent coherent states to be fitted and reduces the choice of phase to either zero, or $\pi$. Any further knowledge should generally be used to reduce the randomness of the positions of the coherent states to be fitted to $|\Psi\rangle$. The semiclassical considerations in the following section may be a further guideline. When this knowledge is exhausted, the best course is to optimize random guesses by Monte Carlo fitting of arbitrary gaussians, within the given constraints.

Specifically, in order to measure the quality of the fitting, we define the relative error

$$
\sigma(x, A, N)=\sum_{l=1}^{M}\left(\rho_{\psi}\left(x_{l}\right)-\sum_{k=1}^{N} A_{k} G_{k}\left(x_{l}\right)\right)^{2} / \sum_{l=1}^{M} \rho_{\psi}^{2}\left(x_{l}\right)
$$

as the mean square deviation between the known Husimi function $\rho_{\psi}(x)$ of a quantum state $|\psi\rangle$ and its fitting to the superposition of gpps defined in (9). This error $\sigma$ has to be minimized for a set of $M$ grid points $x_{l}=\left(p_{l}, q_{l}\right)$. To fit this superposition of gpps as closely as possible to $\rho_{\psi}(x)$, we proceed as follows: (i) the centers $x_{k}=\left(p_{k}, q_{k}\right)_{k=1, . ., N}$ of the coherent states are obtained by Monte-Carlo sampling the region where the distribution is significant; that is the region where the distribution exceeds a certain threshold $\delta$; (ii) the centers must not be too close, this means a minimum distance $\Delta$ between centers is fixed a priori, thereby avoiding overlaps and reducing the total number $N$ of gpps used for the representation; (iii) starting with $N=1$ we increase $\mathrm{N}$ by one and repeat the process until the desired accuracy is reached. Since only the coefficients $A_{k}$ are fitted, minimizing the error $\sigma$ reduces to a problem of linear optimization which is equivalent to solving the real matrix equation

$$
G A=\rho_{\psi}
$$

where the phase space points $x_{l}=\left(p_{l}, q_{l}\right)_{l=1, . ., N}$ that define the matrix elements $G_{l k}=G_{k}\left(x_{l}\right)=\exp \left(-\frac{\left(x_{l}-x_{k}\right)^{2}}{2 \hbar}\right)$ are randomly selected. Here, $A=\left(A_{1}, A_{2}, \ldots, A_{N}\right)$ contains the coefficients to be determined and $\rho_{\psi}=\left(\rho_{1}, \rho_{2}, \ldots, \rho_{N}\right)$ those of the known Husimi function. In general, the matrix $G$ is expected to be sparse and a variety of special algorithms can be used to solve (26) efficiently [21]. In principle one may also consider to optimize this fitting with respect to the widths of the gpps; but since the Husimi function is always smooth and positive definite, it is convenient to keep them frozen. In what follows, we have tested our method on the fitting of the eigenstates of the harmonic oscillator (HO) and that of the Morse oscillator (MO) supporting 18 

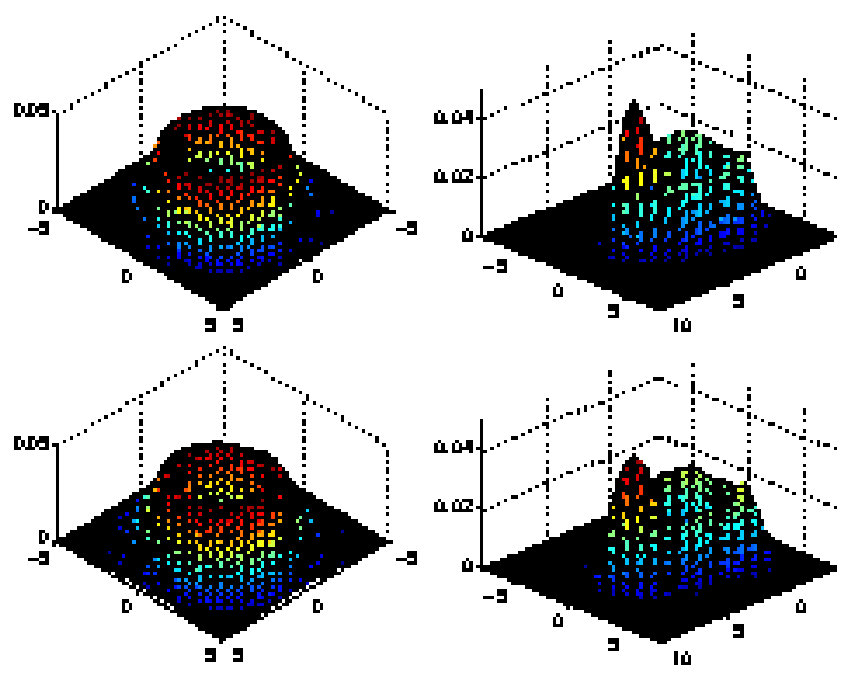

Figure 1. Husimi distributions of the 3rd excited state of the harmonic oscillator (HO) and for the 5th excited state of the Morse oscillator (MO); (top : original, bottom: fit). The fitting of HO (left) is reproduced with $N=7(\delta=0.035, \Delta=0.5)$ and that of MO (right) with $N=14(\delta=0.02, \Delta=1.3)$.

bound states [22]. We have not used the knowledge that these states have time reversal symmetry in the way mentioned earlier, so we should obtain results of comparable quality even for states with complex wave functions. Figure 1 shows, on the top, the known Husimi function for the 3rd excited state of $\mathrm{HO}$ (left) and for the 5th excited state of $\mathrm{MO}$ (right), with parameters $\omega=m=\hbar=1$. On the bottom the corresponding fitted distributions are shown. We found that $N=7$ is sufficient to reproduce quite well the Husimi function of $\mathrm{HO}$ whereas 14 coherent states (gpps) are needed in the MO example, to achieve a global relative error of $\sigma \leq 0.01$. The respective values for the threshold $\delta$ and the minimum distance $\Delta$ are given in the caption of figure 1 . This preliminary fit on the Husimi function provides us with the total number $N$ of gpps, their location in phase space $\left(p_{k}, q_{k}\right)$ and the expansion coefficients $A_{k}$. These coefficients are related to those of the coherent states $a_{k}$ and their phases $\theta_{k}$ by

$$
a_{k}=\sqrt{2 \pi \hbar A_{k}} \exp \left(i \theta_{k}\right) .
$$

Up to this point, the phases $\theta_{k}$ are still unknown. We only need to determine the phases differences $\theta_{j k}$ in (15) which can be easily extracted from (24). This says that, for $N$ gpps, $N(N-1) / 2$ chords $\left(\theta_{j k}\right)$ are required to reproduce the Wigner function. These $N(N-1) / 2$ pairs of gaussians (chords) are symmetrically spaced about $\xi=0$, so that, being more dense, they overlap more than the original set of $N$ gaussians in phase space. Figure 2 shows on the top the Wigner function of the 3rd excited state of the HO together with its corresponding fit obtained with 7 gpps. The present fitting reproduces the Wigner function everywhere in phase space, even for negative regions which are signatures of quantum interference. This is clearly illustrated in figure 3 where 


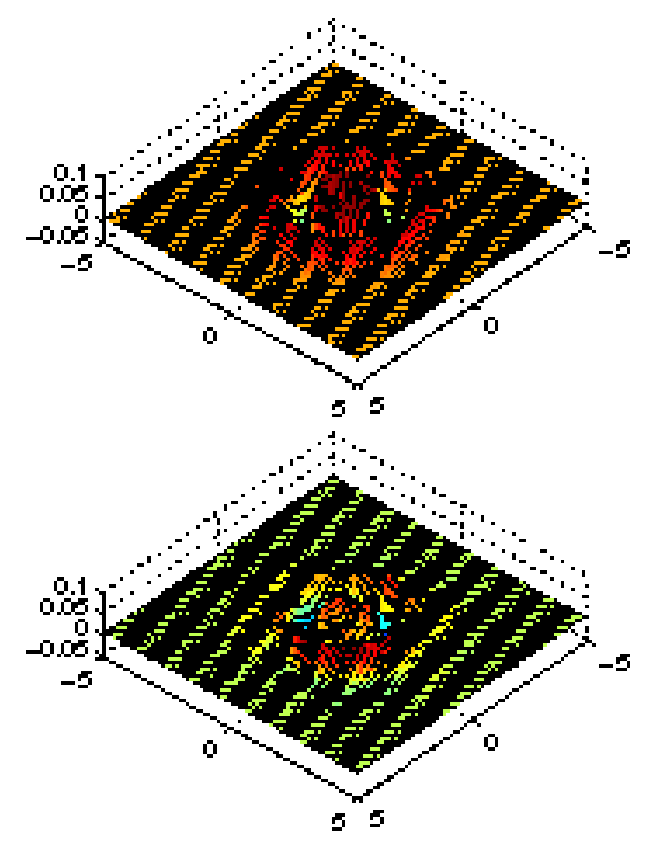

Figure 2. Wigner distributions of the 3rd excited state of HO (top) and its corresponding fit (bottom) with $N=7$ as in figure 1 .

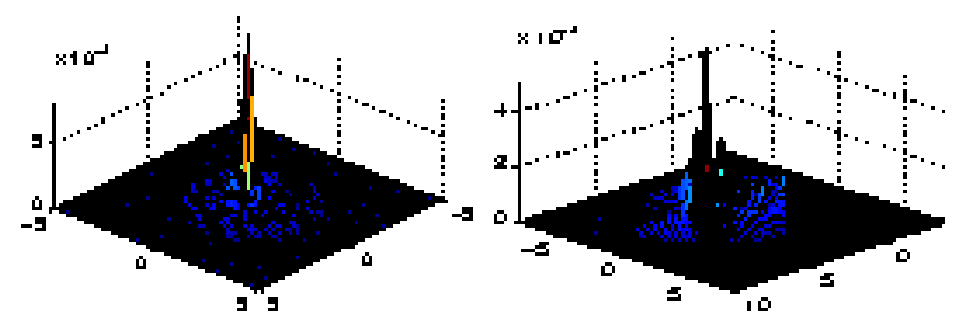

Figure 3. Deviations of the Monte Carlo Wigner sampled of the 3rd excited state of HO (left) and of the 5th excited state of MO (right).

the deviations of the Monte Carlo sampled Wigner function, less than $10^{-4}$ everywhere in phase space, are depicted for both, the HO (left, $n=3$ ) and the MO (right, $n=5$ ). The global relative error $\sigma$ is subsequently found not to exceed 0.01. Moreover, we have computed the number $N$ of gpps needed to represent the Wigner functions of the eigenstates of our test systems, as can be seen in figure 4 . One sees that $N$ grows proportionally to $\sqrt{n}$ as the vibrational quantum number $n$ increases. In the following section, we provide a semiclassical justification of this result by means of the WKB quantization.

\section{Semiclassical approach}

A semiclassical state $|\Psi\rangle_{S C}$ is supported by a curve in phase space, in the case of one degree of freedom, or in general by a surface with half the phase space dimension [16, 17. 


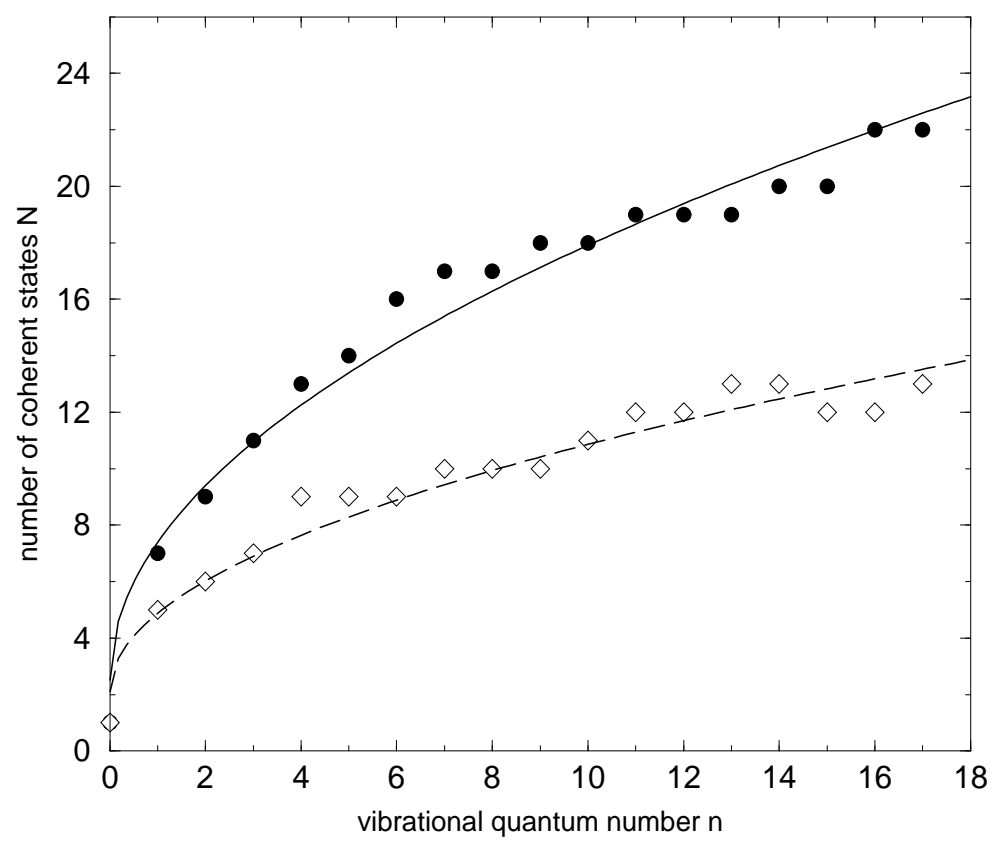

Figure 4. Number of coherent states $N$ used for the fitting versus the vibrational quantum number $n$ for both, harmonic oscillator $(\diamond$, dashed line: fit with $N=$ $2.77 \sqrt{n}+2.10)$ and Morse oscillators $(\bullet$, solid line: fit with $N=4.87 \sqrt{n}+2.51)$.

If the curve or surface is closed, it must be Bohr-quantized and it will be symmetric about $p=0$ in the case of time reversal symmetry. In the case of the position representation, for each branch of the curve (surface), $p_{j}(q)$, one defines the action

$$
\mathcal{S}_{j}(q)=\int_{q_{0}}^{q} p_{j} d q,
$$

leading to the generalized WKB wavefunction,

$$
\langle q \mid \Psi\rangle_{S C}=\sum_{j} a_{j}(q) \exp \left(\frac{i}{\hbar} \mathcal{S}_{j}(q)\right) .
$$

The amplitudes $a_{j}(q)$ can also be expressed in terms of the actions, but the important point is that they are purely classical so that the only $\hbar$-dependence occurs in the exponential. Thus locally, for any small range of positions, the semiclassical wave function reduces to a superposition of plane waves characterized by the wave-vectors $p_{j}(q) / \hbar$. The semiclassical Wigner function is also defined in terms of an action $\mathcal{S}_{j}(x)$ with respect to the classical curve (surface). But instead of the area between the curve and the $q$-axis, we are now concerned with the area sandwiched between the curve and one of its chords. The latter, $\xi_{k}(x)$, is selected by the property that it is centred on the point $x$, as shown in figure 5. Thus, for one degree of freedom [18],

$$
W_{\Psi}(x)_{S C}=\sum_{k} A_{k}(x) \cos \left(\frac{\mathcal{S}_{k}(x)}{\hbar}-\frac{\pi}{4}\right),
$$




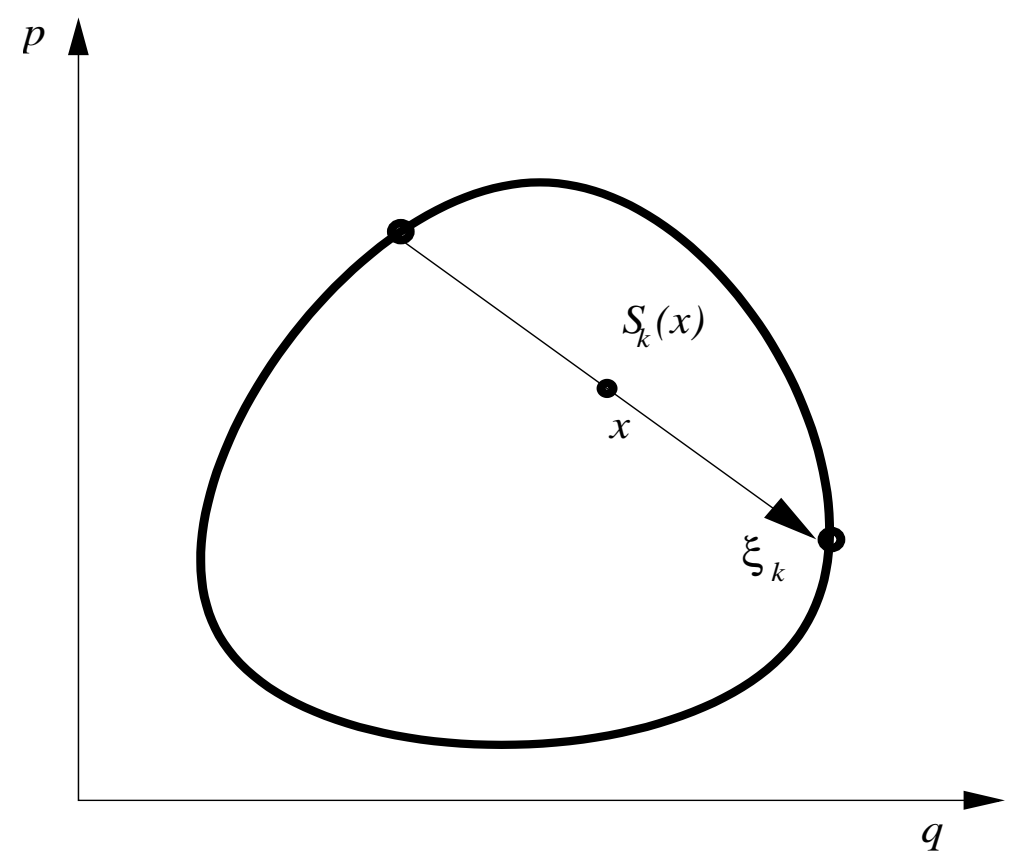

Figure 5. Geometrical illustration of the chord $\xi_{k}(x)$ in phase space $x(p, q)$.

with straightforward generalizations [19]. The important point is that [15]

$$
\frac{\partial \mathcal{S}_{k}}{\partial x}=\mathbf{J} \xi_{\mathbf{k}}
$$

where we use the symplectic matrix $\mathbf{J}$ defined in (5). Thus the semiclassical state is again represented by a superposition of waves,

$$
W_{\Psi}(x)_{S C} \approx \sum_{k} A_{k}(x) \cos \left(\frac{\delta x \wedge \xi_{k}(x)}{\hbar}-\frac{\pi}{4}\right),
$$

but now these are phase space waves. Comparison of this expression with (22) immediately reveals that these waves have exactly the same wave vector as a pair of gaussians at the tip of each chord $\xi_{k}(x)$. It follows that an important feature of a semiclassical state is automatically reproduced by fitting it with gaussians precisely placed on the corresponding classical curve (surface). Notice that, in fact, this is a major a priori obstacle to performing the fitting, because the oscillations near the midpoint of a long chord are very fine for $\hbar<<\mathcal{S}_{k}$. One would need very narrow gaussians indeed to fit these phase space waves directly, rather than having them arise naturally as interferences. All that is left to determine the phase: $\mathcal{S}_{k} / \hbar-\pi / 4$. How can we single out the region in which to place the gpps, if we have no a priori knowledge of the classical structures correponding to a quantum state? The obvious course is to smooth the Wigner function with a Gaussian window. In other words, we initially fit the Husimi function. Recalling that the gpps have linear width of order $\sqrt{\hbar}$, while the wavelength of the Wigner oscillations are $\hbar /\left|\xi_{k}\right|$, it follows that this gaussian window erases effectively 
a semiclassical Wigner function, except in the limit of very small chords. This is a simple explanation of the well known fact that only in the neighbourhood of the classical curve (surface) itself is the semiclassical Husimi function appreciable. In the simple case of one degree of freedom, the Husimi function is concentrated near the energy shell. Thus, by fitting gpps to a Husimi function, one automatically samples its relevant classical mainifold, if it happens to have a (possibly unknown) semiclassical structure. A basic assumption in the above argument is that the gpps along the curve (surface) are neither too crowded, to avoid confusing superpositions, nor too sparsely spaced, lest gaps should arise in the fitting. It is thus easy to estimate the growth of the number of coherent states required to fit the eigenstate $|n\rangle$ with the degree of excitation. Since the linear width of the gaussian scales as $\sqrt{\hbar}$, the phase space area of the curve grows as $n \hbar$ and hence its length grows as $\sqrt{n \hbar}$, the number of equispaced gaussians needed to cover the curve grows as $\sqrt{n}$. Of course, we have assumed here that the shape of the curve does not change with $n$, as for the harmonic oscillator. If the eigencurve elongates for higher excitation, the number of gpps necessary for a good fit will grow as $n^{\alpha}$, with $1 / 2 \leq \alpha \leq 1$. We can also estimate the growth in time of the number of gpps required to fit an evolving semiclassical state with arbitrary precision. According to the theory of van Vleck [16, it is sufficient to evolve the curve (surface) classically and then to reconstruct the wave function. This principle can also be applied to Wigner functions, according to Berry and Balazs [20. If the driving Hamiltonian is chaotic, then the curve will stretch at a rate depending on the Lyapunov exponent, $\lambda$. Clearly, this also determines the initial rate of growth of the number of gpps needed for an adequate fit. Ultimately, when the curve covers densely all the available phase space (the energy shell of the driving Hamiltonian), a steady state will be reached where the number of gpps saturates.

\section{Conclusions}

Optimal fitting of quantum states by phase space gaussians is achieved by first fitting the Husimi function and then determining the quantum phases from the chord function, i.e. the Fourier transform of the Wigner function. It is clear from the discussion in the introduction that the density of gaussians in phase space must be finely adjusted: If ther are too few, essential features of the state will be missed, whereas an excessive number of gaussians would introduce interference terms in the Husimi function itself, which could only be accomodated by a much more complex variation of this method. The numerical examples in section 4 indicate clearly that, as well as achieving excellent overall accuracy, all essential qualitative features of the states are captured by this method, using a basis of gaussians that grows more slowly than the excitation number of the fitted states. This last result and further insight into the fitting procedure, follows from the semiclassical analysis in the previous section. This can be generalized readily to states of quantum systems with higher degrees of freedom if these are eigenstates of integrable systems (i. e. if they are supported by a Lagrangean surface in phase 
space; see e. g. [17]). Of course, more gaussians will then be needed for the fitting, but the preliminary fit of the Husimi function should still provide optimal results. The potential for this method to deal with the eigenstates of chaotic systems is even more interesting. Though the chord and centre description of the Wigner function still applies to mixtures of eigenstates over narrow energy windows, no classical theory accounts for individual eigenstates at present. Therefore, it will be extremely useful to describe these as interfering superpositions of gaussians placed near the energy shell. Of course, the size of this basis would diverge at the classical limit as $\hbar \rightarrow 0$, but manageable approximations should be attainable for finite excitations.

\section{Acknowledgments}

AK gratefully acknowledges the financial support by Alexander von Humboldt (AvH) Foundation/Bonn-Germany, under the grant of Research fellowship No.IV.4-KAM 1068533 STP. AMOA thanks the MPIPKS-Dresden for a Martin Gutzwiller Fellowship, during which this work was initiated, and acknowledges support from CNPq and Pronex in Brazil.

\section{References}

[1] Horenko I, Schmidt B and Schuette C 2002 J. Chem. Phys. 1174643

[2] Johansen L M 1998 Phys. Rev. Lett. 805461

[3] Lutterbach L G, Davidovich L 1997 Phys. Rev. Lett. 7825471997 Wax A, Bali S, Thomas J S 1999 Opt. Lett. 24 1188; Lee K F, Reil F, Bali S, Wax A, Thomas J E 1999 Opt. Lett. 24 1370; Nogues G, Rauschenbeutel A, Osnaghi S et al 2000 Phys. Rev. A 62054101

[4] Cohen L 1995 Time-Frequency Analysis. New York: Prentice Hall, 113.

[5] Bartelt H O, Brenner K H and Lohmann A W 1980 Opt. Comm. 3232

[6] Riley M and Bolton J S 1993 Speech Time-Frequency Representations. Kluver Academic Publishers.

[7] Meng Q and Qu L 1991 Mechanical systems and signal Proceessing 5155

[8] Cohen Tannoudji C, Diu B and Laloe F 1977 Quantum Mechanics, Wiley, New York

[9] Husimi K 1940 Proc. Phys. Math.Soc.Japan 22264

[10] Radmore P M and Barnett S M 1997 Methods in theoretical quantum optics, Oxford Univ Press

[11] Wolfgang P Schleich 2001 Quantum Optics in Phase Space, Wiley VCH, Berlin

[12] Wigner E P 1932 Phys. Rev. 40749

[13] Moyal J E 1949 Proc. Camb. Phil. Soc. Math. Phys. Sci. 4599

[14] MarksII R J, Walkup J F and Krile T F 1977 Appl. Opt. 16746

[15] Ozorio de Almeida A M 1998 Phys. Reports 295265

[16] Van Vleck J H 1928 Proc. Math. Acad. Sci. USA 14178

[17] Ozorio de Almeida A M 1988 Hamiltonian Systems: Chaos and Quantization Cambridge University Press, Cambridge

[18] Berry M V 1977 Phil. Trans. R. Soc. 287237

[19] Ozorio de Almeida A M and Hannay J H 1982 Ann. Phys., NY Ann. 138115

[20] Berry M.V. and Balazs 1979 J. Phys. A: Math. Gen. 12625

[21] Press W H, Teukolsky S A, Vetterling W T and Flanneryt B P 1992 Numerical recipes in Fortran 22

[22] Kenfack A and Rost J M 2003 unpuplished 